\title{
Barchan dunes on Pluto?
}

\author{
Eric J. R. Parteli ${ }^{1, \star}$ and Thorsten Pöschel ${ }^{2, \star \star}$ \\ ${ }^{1}$ University of Cologne, Cologne, Germany \\ ${ }^{2}$ University of Erlangen-Nuremberg, Erlangen, Germany
}

\begin{abstract}
We show that the orientation and morphology of bedforms occurring on top of Pluto's smooth ice coats are consistent with an aeolian origin under conditions of unidirectional flow. From scaling relations for dune size as a function of attributes of atmosphere and sediments, we find that the average diameter of the granular particles constituting such bedforms - assuming an aeolian origin — lies within the range $600 \mu \mathrm{m}<$ $d<750 \mu \mathrm{m}$. Our findings show that, owing to the effect of hysteresis in the minimal threshold wind velocity for saltation, dune migration on Pluto can occur under wind speeds that are common to Earth and Mars.
\end{abstract}

\section{Introduction}

Since New Horizon's encounter with Pluto in July 2015 we know that Pluto has a geologically active surface consisting of dark, cratered terrains alternating with huge icy mountains and flat, bright soils free of impact craters [1]. Several processes have been suggested to explain the complex topography, in particular chemical reactions triggered by ultraviolet radiation from the sun, heterogeneous rates of ice sublimation and deposition induced by gradients of surface temperature, and resurfacing due to radioactive heating from within the planetary body [2]. In this paper, we consider the role of wind as a geological process on Pluto. Based on images sent by New Horizons, we identify surface features on the surface of Pluto that resemble very much aeolian dunes on Earth and Mars. In particular, the referred bedforms display a dependence of shape on the size which is inherent of aeolian dunes. As we will show here, assuming an aeolian origin for these bedforms, theory that successfully describes the process of dune formation on Earth and Mars can be applied to constrain formative wind speeds and average grain sizes on Pluto [3-5].

\section{The barchans}

Bedforms with morphology similar to aeolian dunes of Earth and Mars occur on top of Pluto's bright, ice-coated crater-free surface, between Norgay and Hillary Montes (Fig. 1). Some of the bedforms display a dome-like shape while others resemble crescent-shaped barchan dunes which form under unidirectional winds, in areas of limited sand supply, and have two limbs pointing in the wind direction [3-9]. The orientation of the barchan limbs indicates, thus, the prevailing sediment-moving wind trend,

\footnotetext{
${ }^{\star}$ e-mail: eric.parteli@uni-koeln.de

${ }^{\star}$ e-mail: thorsten.poeschel@fau.de
}

denoted by the dashed arrow in Fig. 1c (see Fig. 3 for reference).

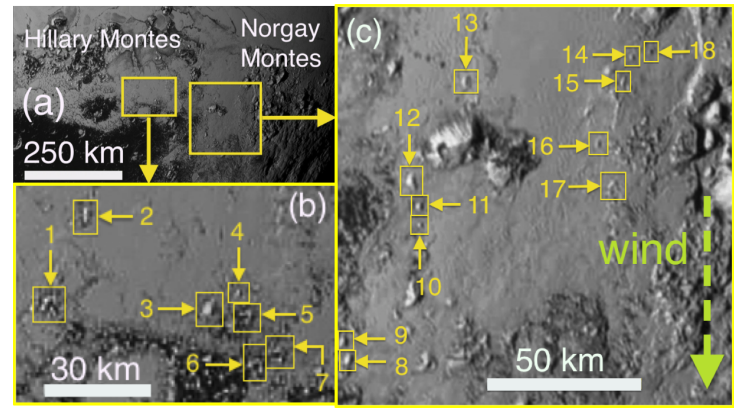

Figure 1. Dunes on the thin ice sheet between Hillary Montes and Norgay Montes on Pluto. The dunes occur within the areas enclosed by the rectangles in (a). These areas correspond to images (b) and (c), where each dune, enclosed by a box, is identified by a number for reference in the plot of Fig. 2. The dashed arrow in (c) denotes the mean formative wind direction, which is obtained from the orientation of the limbs of the barchan dunes (see Fig. 3). Images credit: NASA/JHUAPL/SWRI.

Figure 2 shows that the along-wind width $L$ and crosswind width $W$ of the bedforms correlate linearly, which is the same behavior of barchan dune fields on Earth and Mars [3-5]. Some barchans display an asymmetric shape with one limb elongated downwind, which is common feature of dunes on Earth and Mars caused by wind trend fluctuations, local topography and inhomogeneous influx conditions due to the presence of upwind dunes $[8,9]$.

\section{Model for aeolian transport on Pluto}

The presence of dunes implies that winds on Pluto have occasionally exceeded the minimal threshold to transport granular materials - most probably constituted of nitrogen 


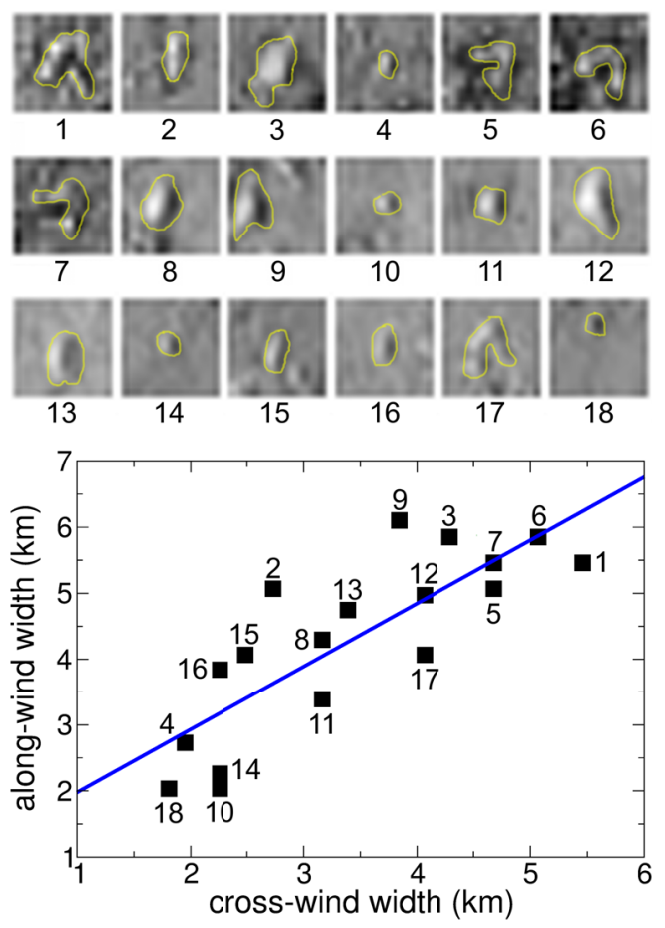

Figure 2. Bedform's morphometry. Left: dunes 1-18 from Fig. 1. The contour line was added in each image in order the highlight the dune shape. Each image is $\approx 9 \mathrm{~km}$ across. Right: alongwind and cross-wind widths ( $L$ and $W$, respectively) of the imaged dunes (squares). The best fit using the equation $L=a+b \cdot W$ yields $a \approx 1.02 \mathrm{~km}$ and $b \approx 0.96$ (continuous line).

ice particles $[1,2,10,11]$ - along the surface. As shown in Ref. [5], natural grains occurring in dune fields have a broad range of sizes, and the transport mode depends on the particle size. While gravels, that is particles larger than $1 \mathrm{~mm}$, are too heavy to enter the aeolian transport layer and thus roll or slide (creep) on the surface, dust particles, which are smaller than about $60 \mu \mathrm{m}$, are transported by the wind through suspension. However, particles in the intermediate range move mainly through saltation, which is the particle transport mode leading to dune formation and consists of grains moving in nearly ballistic trajectories and ejecting new particles upon collision with the soil [6].

Wind speeds 100 times larger than those shaping terrestrial dune fields are needed to initiate sediment transport on Pluto (whereas this difference is mainly due to the 10,000 times smaller atmospheric density of Pluto [12], compared to the Earth's). However, recent theory of aeolian sediment transport shows that the effect of hysteresis in the threshold wind speed for saltation transport [13-16] - which means that, once initiated, saltation can be sustained at much smaller wind speeds - is much stronger under rarified atmospheres. On Earth, the threshold for saltation maintenance $\left(u_{t}\right)$ is about $80 \%$ the threshold for saltation initiation $\left(u_{\mathrm{ft}}\right)$, while on Pluto $u_{\mathrm{t}}$ is $10-20$ times smaller than $u_{\mathrm{ft}}$ (see Fig. 4). In other words, dunes might form and migrate on today's Pluto as long as surface wind speeds on this planetary body occasionally exceed values

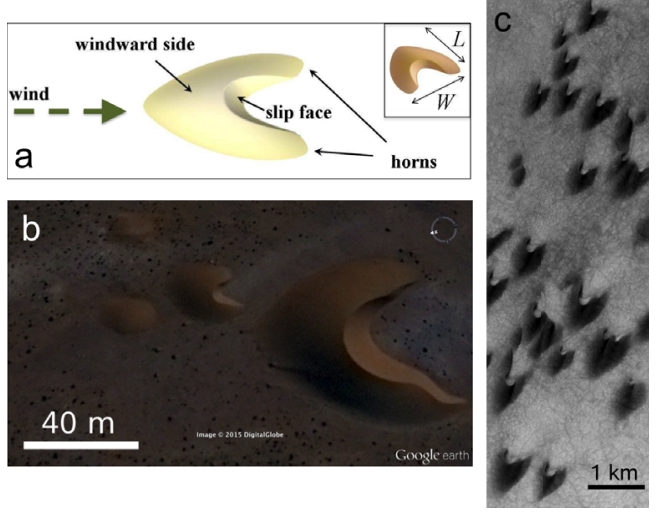

Figure 3. The barchan dune shape. a, Schematic diagram of a barchan dune. The dashed arrow indicates the prevailing wind direction. The definitions of along-wind width $L$ and cross-wind width $W$ of a barchan are provided in the inset. Sediments are transported downwind along the windward side of the barchan through saltation, thereby accumulating on the dune crest. As the amount of sediments on the crest increases, the dune's leeward side becomes increasingly steeper. When the slope of this side exceeds the angle of repose of the granular material (which for terrestrial and martian sand is about $34^{\circ}$ ), the downwind surface of the dune relaxes through avalanches in the direction of the steepest descent - a slip face forms. Furthermore, since the downwind migration velocity of aeolian bedforms scales with the inverse of the bedform size $[6,7,18]$, the dune flanks which are the lowest parts of the dune - appear advanced downwind relative to the central part of the dune, thus giving rise to the limbs that are characteristic of the barchan shape. b, barchan dunes in Morocco, near $26^{\circ} 5^{\prime} \mathrm{N}, 13^{\circ} 2^{\prime} \mathrm{E}$ (image credit: Google Earth); c, barchan dunes in Arkhangelsky crater, Mars, near $41^{\circ} 4^{\prime} \mathrm{S}, 24^{\circ} 8^{\prime} \mathrm{W}$ (image credit: NASA/JPL/MSSS). It can be seen that limbs are absent from barchans smaller than a minimal threshold size, which on Earth and Mars is about $10 \mathrm{~m}$ and $200 \mathrm{~m}$, respectively. The same behavior is observed on Pluto (see Figs. 1 and 2).

that are not uncommon to dune fields of our planet. Mechanisms for transport initiation on Pluto are still uncertain. Candidates might be sporadic gusts of much stronger aeolian activity or particle lifting from the soil due to insolation-induced convective gas flows within the granular bed - which, as demonstrated recently [17], is a relevant direct particle entrainment process under conditions of low atmospheric pressure.

The fact that barchan limbs are absent from all bedforms of cross-wind width smaller than a threshold size $W_{\min }$ (which, from Fig. 1, is of typical size $3 \mathrm{~km}$ ) is, indeed, a feature characteristic of dunes that migrate on top of bedrock under constant wind direction [5, 18-20]. This minimal dune size scales with the characteristic length needed by the mass flux of particles in saltation to adapt to a change in flow conditions -- the flux saturation length, $L_{\text {sat }}$ (see Refs. $[13,18,21]$ ). In particular, the saturation length dictates the transient distance needed for the flux to adjust to a topography-induced change in the average turbulent surface wind shear stress. Since the downwind position of the maximal wind shear stress over sand 


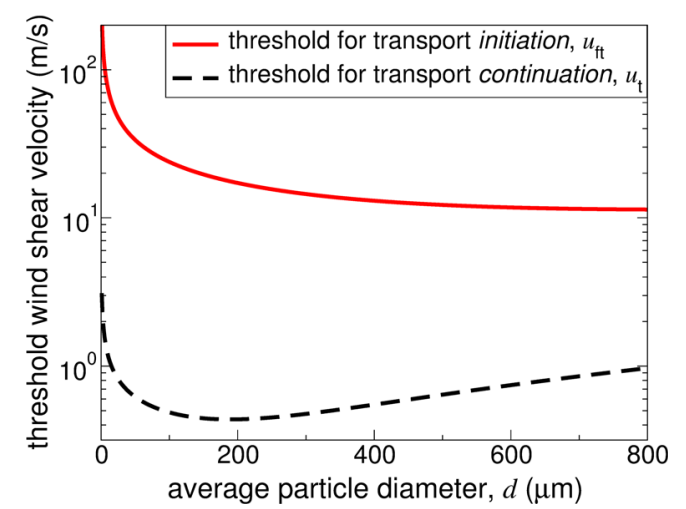

Figure 4. Minimal threshold wind shear velocity for saltation on Pluto, as a function of average particle diameter. The threshold for transport initiation, $u_{\mathrm{ft}}$, is computed using the model of Ref. [24], while the threshold for transport continuation, $u_{t}$, is obtained using the model of Ref. [16]. The results correspond to average surface pressure of $20 \mu \mathrm{bar}$ and temperature $44 \mathrm{~K}$, assuming atmosphere composed mainly of $\mathrm{N}_{2}$.

hills and domes is shifted upwind of the bedform's crest $[18,20,22]$, the development of a sand heap into a barchan occurs only if $L_{\text {sat }}$ is smaller than this upwind shift, thus causing net deposition on the crest and bedform growth. It was shown [5] that this condition is fulfilled for bedforms that are larger than about $12 \times L_{\text {sat }}$. Because $L_{\text {sat }}$ is a function of the attributes of sediment, fluid and wind power $[13,18,21]$, the value of $W_{\min }$ of the bedforms imaged by New Horizons poses one constraint to the physical conditions causing sediment transport and dune formation on Pluto.

Specifically, the scale of the minimal dune on Pluto provides a proxy for the range of average grain size $d$ and wind shear velocity $u^{*}$ - which is proportional to the mean flow velocity gradient in turbulent boundary layer flow [23] -- leading to dune formation under atmospheric conditions valid for today's Pluto. This range is obtained from the equation $12 L_{\mathrm{sat}}\left(u^{*} / u_{t}, d\right)=W_{\min }$, where $L_{\text {sat }}$ is computed with the model of Ref. [13] and the minimal threshold for sustained transport, $u_{t}$, is a function of the atmospheric density, particle diameter and density, air viscosity and gravity $[13,16]$. The main plot of Fig. 5 shows the values of $d$ and $u^{*} / u_{t}$ which satisfy the equation above (continuous line).

We can now constrain approximate value of wind speeds and grain sizes for the barchan-like bedforms as explained in the following. Since dunes-forming $u^{*}$ values are upper-bounded by the minimal threshold for suspension -- which is about $4 u_{t}$ (see Ref. [21]) and is indicated by the dotted horizontal line in Fig. 5 - and given the prohibitively large values of the minimal threshold shear velocity for direct entrainment of powder-sized particles of diameter smaller than $20 \mu \mathrm{m}$ (for which cohesive forces are large compared to particle weight and aerodynamic lift [24-26]), we estimate that the most likely diameter range of the sediments composing Pluto's bedforms is, based on the main plot of Fig. 5, $600 \mu \mathrm{m} \leq d \leq 750 \mu \mathrm{m}$. This range

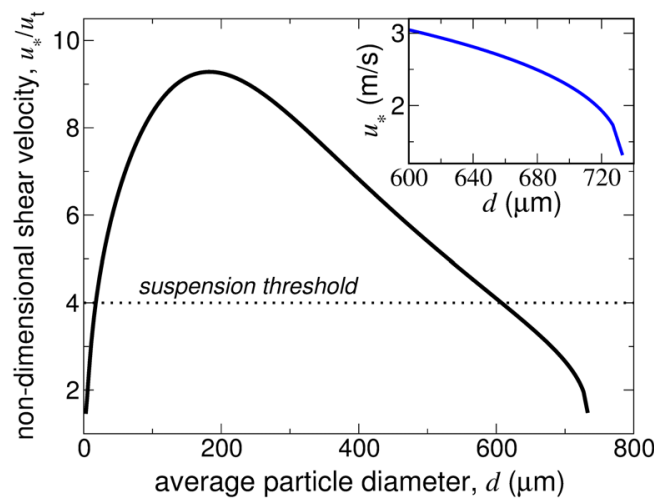

Figure 5. Range of formative wind shear-velocities and grain sizes from the scale of Pluto's dunes. The continuous line in the main plot denotes the solution for $u^{*} / u_{t}$ and $d$ obtained from the equation $12 L_{\mathrm{sat}}\left(u^{*} / u_{t}, d\right)=W_{\min }$, where the saturation length of sediment transport, $L_{\mathrm{sat}}$, is computed with the model of Ref. [21] and $W_{\min } \approx 3 \mathrm{~km}$ is the minimal barchan dune size on Pluto. Moreover, $u_{t}$ is obtained as a function of the attributes of sediments and atmosphere with the model of Ref. [16]. The suspension threshold $u^{*} / u_{t}=4$ is indicated by the dotted horizontal line $[13,16]$. Based on the main plot, the most plausible range of shear velocity $u^{*}$ and grain size $d$ leading to dune formation on Pluto is shown in the inset.

corresponds to particle sizes of coarse sand [6]. The corresponding range of shear velocities leading to the dune scale on Pluto is $1.5 \mathrm{~m} / \mathrm{s} \leq u^{*} \leq 3.0 \mathrm{~m} / \mathrm{s}$ (inset of Fig. 5), which is comparable to the wind speeds required to initiate sand transport on Mars [27] and is about one order of magnitude larger than values of wind shear velocity of desert dune fields on Earth [28].

We have thus applied theory of sediment transport, which has proven to successfully describe dune formation in a broad range of physical environments (including Venus, Earth and Mars) [3, 13, 15, 16, 18, 21, 25], to estimate possible values of average grain size and wind speed formative of crescent-shaped bedforms occurring between Norgay and Hillary Montes on Pluto. In particular, the theory accounts for the stronger hysteresis in the threshold for saltation under rarified atmospheres (compared to the Earth). From the scale of the bedforms in Fig. 1, the theory predicts they are constituted by (coarse) sand-sized particles and were formed by wind speeds that are typical of Martian storms. Moreover, it is clear that betterquality images would be necessary to clarify the details of these bedforms, in spite of the resemblance of their crescent shape to barchan dunes on Earth and Mars. However, there is one further and stronger argument in favor of aeolian origin of the referred bedforms, that is the dependence of their shape (morphometry) on the size follows the same behavior of barchans on Earth and Mars (in particular the existence of a minimal size). Nevertheless, it remains uncertain how these bedforms could form, that is what is the origin of the sediments?

Possible sources for dune sediments, which have been identified in a previous discussion [10], include impact ejecta, as well as fine component of regolith impact; erup- 
tive plume ("cryoclastic") fallout -- on Mars, the particle diameter of dunes sand, which is of volcanic origin, is most probably within the range between $100-600 \mu \mathrm{m}$ $[25,29]$, thus smaller than the value obtained here for Pluto's dunes; atmospheric precipitation (snow), and abrasion of bed-ice surfaces. For the barchan dunes investigated here, one particular aspect in favor of last factor is the proximity of the dunes to the icy mountains at the dune's upwind area. Clarifying the origin of the sediments might help constrain the expected range of grain sizes which has been predicted from our calculations.

Whatever the origin the sediments, the present manuscript has adopted the assumption of an aeolian origin for the bedforms in Fig. 1, based on the observations described previously. However, despite the above discussion, it is pertinent question whether other processes but aeolian transport may have contributed to generate the sediments or even to shape these bedforms. While previous works already recognized the possible role of aeolian transport for shaping Pluto's surface, here we have shown how theory can be applied to obtain information on grain size and wind speed from the scale of dunes on Pluto. The method presented here should be now applied to other candidate dune fields at other places on Pluto. Our calculation results remain to be confirmed by data obtained by another approach, such as wind tunnel experiments of aeolian transport under conditions of pressure and temperature valid for Pluto.

While the present estimate of particle diameter is based on well-established theoretical expressions, which have proven to successfully predict the minimal dune size over 5 orders of magnitude in grain to fluid density ratio [18], it relies on the assumption that Pluto's bedforms have been formed under the average atmospheric density of today's Pluto. We note that global circulation models of Pluto have brought useful insights about wind behavior at heights larger than $\sim 200 \mathrm{~m}$ [30], and it would be interesting to compare our predicted range of average surface wind speeds with results from a mesoscale atmospheric model that resolves local topography. As shown recently [11], Pluto's surface displays a broad range of landform types and a diversity of terrain ages, and thus the quantitative investigation performed here shall provide one useful means for constraining possible origins of different types of aeolian bedforms to be imaged by New Horizons in the months and years ahead.

\section{Acknowledgements}

We acknowledge the German Research Foundation (DFG) for funding through its Cluster of Excellence "Engineering of Advanced Materials", ZISC, FPS, at the University of Erlangen-Nuremberg. E.J.R.P. acknowledges financial support from DFG through Grant RI 2497/3-1.

\section{References}

[1] https://www.nasa.gov/mission_pages/newhorizons/ main/

[2] A. Witze, Nature, doi:10.1038/nature.2015.17986 (2015).

[3] E. J. R. Parteli, O. Durán and H. J. Herrmann, Phys. Rev. E 75, 011301 (2007).

[4] R. A. Bagnold, The Physics of Blown Sand and Desert Dunes (Methuen, London, 1941).

[5] K. Pye and H. Tsoar, Aeolian Sand and Sand Dunes (Springer, Berlin, 2009).

[6] H. J. Finkel, J. Geol. 67, 614 (1959).

[7] S. L. Hastenrath, Z. Geomorphol. 11, 300 (1967).

[8] M. C. Bourke, Icarus 205, 183 (2010).

[9] E. J. R. Parteli et al., Aeolian Research 12, 121 (2014).

[10] J. M. Moore, et al, Icarus 246, 65 (2015).

[11] S. A. Stern, et al., Science 350, 6258 (2015).

[12] C. B. Olkin, et al., Icarus 246, 220 (2015).

[13] T. Pähtz, J. F. Kok, E. J. R. Parteli and H. J. Herrmann, Phys. Rev. Lett. 111, 218002 (2013).

[14] M. P. Almeida, E. J. R. Parteli, J. S. Andrade and H. J. Herrmann, PNAS 105, 6222 (2008).

[15] J. F. Kok, Phys. Rev. Lett. 104, 074502 (2010).

[16] T. Pähtz, J. F. Kok and H. J. Herrmann, New J. Phys. 14, 043035 (2012).

[17] C. de Beule, et al., Nature Physics 10, 17 (2014).

[18] K. Kroy, G. Sauermann and H. J. Herrmann, Phys. Rev. Lett. 88, 054301 (2002).

[19] B. Andreotti, P. Claudin and S. Douady, Eur. Phys. J. B 28, 321 (2002).

[20] E. J. R. Parteli, K. Kroy, H. Tsoar, J. S. Andrade Jr. and T. Pöschel, EPJST 223, 2269 (2014).

[21] P. Claudin and B. Andreotti, Earth and Planetary Science Letters 252, 30 (2006).

[22] P. Claudin, G. F. S. Wiggs and B. Andreotti, Boundary-Layer Meteorology 148, 195 (2013).

[23] E. R. van Driest, Journal of the Aeronautical Sciences 23, 1007 (1956).

[24] Y. Shao and H. Lu, J. Geophys. Res. 105, 22437 (2000).

[25] J. F. Kok, E. J. R. Parteli, T. I. Michaels and D. Bou Karam, Rep. Prog. Phys. 75, 106901 (2012).

[26] D. M. Burr, et al., Nature 517, 60 (2015).

[27] R. Greeley and J. D. Iversen, Wind as a Geological Process on Earth, Mars, Venus and Titan (Cambridge University Press, New York, 1985).

[28] S. G. Fryberger and G. Dean, Dune forms and wind regime. In: E. D. McKee (ed.), A Study of Global Sand Seas, no. 1052, Geol. Surv. Prof. Pap. 137 (United States Government Printing Office, Washington, 1979).

[29] K. S. Edgett and P. R. Christensen, J. Geophys. Res. 96, 22765 (1991).

[30] A. M. Zalucha, Monthly Notices of the Royal Astronomical Society 459, 902 (2016). 